\title{
Wearable technology for occupational risk assessment: potential avenues for applications.
}

\author{
I. Pavón ${ }^{1}$, L. Sigcha ${ }^{1}$, P.M. Arezes², N. Costa ${ }^{2}$, G. Arcas ${ }^{1}$, J. M. López ${ }^{1}$. \\ ${ }^{1}$ Instrumentation and Applied Acoustics Research Group (I2A2). ETSI Industriales. Universidad Politécnica \\ de Madrid. Campus Sur UPM. Crta. Valencia, Km 7. 28031 Madrid, ESPAÑA. \\ 2 The Ergonomics \& Human Factors research group (E\&HFg). Universidade do Minho (UMinho) \\ Campus Azurém, 4800-058 Guimarães, PORTUGAL.
}

ignacio.pavon@upm.es

\begin{abstract}
This paper analyzes the opportunities offered by wearable technology for occupational risk assessment. A wide range of electronic devices known as "wearables" include sensors that provide interesting features with the potential to be used in workers' monitoring, sending alert signs (e.g., visual, sound, haptic) and training. In this paper, it has been identified the primary applications in which wearables are being used for risk assessment (motion and activity detection, recognition of work-related musculoskeletal disorders, fall detection, evaluation of exposure to different physical agents, evaluation of exposure to chemical agents, and location of potential hazards). Also, it has been identified the opportunities for improvement in the future using the potential of emerging technologies.
\end{abstract}

\section{INTRODUCTION}

The latest estimate on occupational accidents published by International Labour Organization (ILO) in September 2017, shows that 2.78 million workers die each year from occupational accidents and occupational diseases. Occupational mortality represents $5 \%$ of the world total. The majority of work-related mortality comes from occupational diseases (2.4 million) $86.3 \%$ of the total. The remaining $13.7 \%$ is due to occupational accidents. Work-related injuries and illnesses result in the loss of 3.9\% of world GDP, with an annual cost of 2680 billion $€$. In the case of the EU, the cost of work-related injuries and injuries accounts for 3.3\% of GDP, 476 billion $€$ per year. Occupational accidents and its costs can be significantly reduced by establishing and practicing good occupational health and safety policies (Hämäläinen et al. 2017).

There is an increasing number of technological solutions that use a preventative approach (versus a reactive model), making possible to detect situations of occupational risk before they occur. Using these advanced technologies it is possible to reduce occupational risk and its costs. (Ding et al. 2013)

Information and communications technologies (ICT) can be used to monitor the well-being, progress, and quality of life of someone over a period of time. Devices like smartphones, tablets or wearables (e.g., smartwatches, smart wristbands) are used to manage specific health problems. This technology continues to evolve rapidly, the devices now can communicate with each other taking advantage of the omnipresent internet, and cloud computing services. The combination of the Internet of Things (IoT), Big Data (collection and analysis of large amounts of data) and the smart working environments (SWE) provides an opportunity for monitoring activities conducted by the worker, machinery, and tools (EU OSHA 2017, Li 2018).

Holistic solutions need to be used in managing occupational risk prevention (ORP). Areas for developing and improving include analyzing the inputs and outputs of the system, analyzing the users involved and their needs, improving efficiency and reducing costs, accelerating the risks detection processes and integrating technologies that are currently under development.

Additionally, the resulting synergies should also be considered in managing ORP, such as prevention enhancement, accident and illness reduction, productivity improvement, costs reduction, availability and sharing of information, decision making in quasireal-time, integration with the concepts of digitization of industry (Industry 4.0).

Devices called wearables can be carried on the body or integrated into clothing or other accessories. Wearable's sensors are used to monitor and track the wearer's health, identify the medical needs, and for fitness. Wearables are also used in areas such as entertainment, education, finance, and music (Sazonov et al. 2014, Wright 2014, Page 2015).

There are some initiatives for the use of wearable devices in industrial operations, mainly to the monitoring process and measuring health. Advantages such as portability, energy autonomy, and discrete use have been exploited in several solutions, creating a new paradigm for risk assessment with information shared real-time (Negi et al. 2011, Chan et al. 2012, Kim et al. 2013, Peppoloni 2016, Bieber et al. 2012, Daponte et al. 2013). 
The intelligent wearable devices have processing, data storage, and communication capabilities, which gives them the potential to be a part of integrated systems. Thus, wearables can be used in the field of industrial safety (Podgorski et al. 2017, Bernal et al. 2017, Li 2018).

The aim of this article is to summarize the current state and the main applications of wearable technology related to the ORP. Also, it is presented the opportunities generated by the wearable technology and emerging technologies to improve the current risk management systems. Finally, the foremost challenges and future perspectives of the weareable technology related to ORP will be discussed.

\section{WEARABLE TECHNOLOGY FOR OCCUPATIONAL RISK ASSESSMENT}

The authors searched for published literature using a range of related keywords such as wearable, risk prevention, occupational safety, work monitoring and, risk assessment. The literature was selected according to its relevance with the ORP and with the date of publication after the year 2000.

Based on the literature review have been found numerous solutions that use wearable devices for the management of different occupational hazards. These solutions include motion and activity detection, recognition of work related musculoskeletal disorders, fall detection, evaluation of exposure to different physical agents (e.g., vibrations, noise, thermal environment, and light), exposure to chemical agents, and location of potential hazards.

Recent developments in the areas of environmental intelligence (AmI), the IoT and cyber-physical systems (CPS) have created various solutions for occupational health and safety using smart personal protection devices or smart equipment (Podgorski et al. 2017). These developments emerged from the standardization of low-cost sensors called MEMS (Micro-Electro-Mechanical Systems).

\subsection{Wearables for motion and activity detection:}

One of the primary applications of the wearables is detecting activity and movement. Different studies have used specific wearable devices and commercial devices for their variety of sensors (mainly inertial sensors e.g., accelerometers or gyroscopes) (Yang et al. 2010, Labrador et al. 2013, Mortazavi et al. 2015, Gjoreski et al. 2016).

Also, smartwatches and smartphones have been used simultaneously for the automatic recognition of activities. In (Ramos et al. 2016) the use of two intelligent devices with MEMS inertial sensors shows an increase the accuracy of physical activity recognition. The results show that wearables are a viable alternative to automatic recognition using commercial devices.

For occupational safety and health of construction workers, in (Lee et al. 2017) using a commercial wearable device with several sensors intended for physiological and activity monitoring have made it possible to perform personal measurement on health and well-being of workers. Showing that is possible to identify relationships between health variables to improve the performance, productivity and the safety of workers.

\subsection{Wearables for detection of work-related musculoskeletal disorders:}

For detection of musculoskeletal disorders in the industry, some solutions have been developed mainly using inertial sensors.

In (Peppoloni et al. 2016) a wearable device that analyzes muscle strains and posture have been tested. The usefulness of this device was compared with the traditional method of observational inspection, showing that this type of system can be used in a complete work shift in a discreet way.

Similarly, in (Valero et al. 2017) the analysis of construction workers' postures has been carried out using a network of wireless sensors that identify postures and detect those that may be potentially dangerous. The results suggest that by using this approach, there is an improvement in the productivity and performance of workers employing favorable job positions.

In (Nath et al. 2017, Yan et al. 2017) a similar approach is followed, but using the sensors of several smartphones placed with adapters, in one case on the arms and waist as well on back and neck of construction workers. Showing that it is possible to identify possible ergonomic risks related to the work using two sensors. The systems are presented as a low-cost and efficient solution to prevent works from dangerous positions.

\subsection{Wearables for fall detection:}

Detection of falls has been one of the relevant research topics in recent years. In (Pannurat et al. 2014) a compilatory study of fall detection and prevention systems was conducted using wearable (with inertial sensors) and environmental sensors. The study shows that wearables have some advantages over the environmental sensor systems such as better mobility, easy installation, and have a higher area of use and coverage.

The use of smartphones for fall detection is a growing topic to investigate since these devices have the necessary hardware for fall detection. In the studies of (Habib et al. 2014, Casilari et al. 2015) are 
present solutions for falls detection and prevention by using smartphones and Android devices.

Although smartphones can be considered as an excellent alternative to the dedicated systems, they also present technical challenges in regards to the quality of sensors, power autonomy, and identification of the best smartphone's location on the body.

\subsection{Wearables for assessment of exposure to physical agents:}

Another foremost application for wearable devices is the assessment of exposure to physical agents. One of the relevant topics is the vibration exposure, which can be divided into hand-arm vibration (HAV) and whole body vibrations (WBV).

In (Tarabini et al. 2012) the advantages and disadvantages of the use of accelerometers for the measurement of HAV and WBV were evaluated. This paper concludes that it is possible to design systems that include MEMS accelerometers inside any hand tool, in the operator interface, or inside the seats of automobile structures, tractors, and trucks.

\subsubsection{Hand Arm Vibration (HAV).}

Experiences in the development of HAV meters were carried out by (Morello et al. 2010, Aiello et al. 2012) in which portable devices with MEMS accelerometers were used to measure HAV. These studies are presented as a new approach to safety and risk management, performing in real-time.

Then, in (Liu et al. 2013) a smartphone with a metering application was used as a wearable on the hand. The smartphone was secured to a crafted glove that was placed on a hand, showing a versatile and low-cost solution for risks assessment, train and bring awareness to workers.

Regard the use of smartwatches, in (Pavón et al. 2016) an HAV measurement system based on a commercial smartwatch was developed. Showing that this kind of system can be used in specific vibration risk management tasks due to the operating system restrictions. This study identifies improvement opportunities and presents the advantages (portability, continuous measurement, and cost reduction) of wearable systems over standard measuring devices.

Then, in (Matthies et al. 2016) a system to determinate the exposure to HAV based on classification algorithms were developed using the built-in microphone and accelerometer data. The results indicate some deviation when compared to a reference measuring device. However, using this approach demonstrates that such systems are a viable solution for risk management and monitoring of workers, overcoming the limitations of the device or sensor.

\subsubsection{Whole body vibrations (WBV).}

Smartphones with built-in MEMS accelerometers have been used to measure WBV. In (Cutini et al. 2014) a software application that measures the exposure to vibrations was developed in an Android phone, the system shows deviations in the measurements but presents a simple and low-cost system for carrying out the initial risk assessment and training.

In (Wolfgang et al. 2014 a, b) a WBV metering system was developed in a multimedia device (iPod) and compared with a gold standard vibration analyzer. The results of their experiments suggest that measurements made with the iPod can be used to measure full-body vibrations with minimal error, reducing the costs and complexity to manage this risk.

\subsubsection{Noise.}

In recent years smartphones and mobile applications have been used as a measurement tool for environmental noise and occupational noise assessments. The accuracy of these systems using the built-in microphone have been analyzed in (Kardous et al. 2014, Kardous et al. 2015, Nast et al. 2015, Murphy 2016), the results show variation in the measurements made with these systems.

Then, solutions using smartphones with a highquality external microphone have been tested by (Kardous et al. 2016), in which conclude that the calibration can make the system a useful tool for qualified professionals. Methods for calibration of these systems have been proposed in (Dumoulin 2013, Zhu et al. 2015).

\subsubsection{Occupational thermal environment.}

In (Pancardo et al. 2015) several sensors (accelerometer, humidity, and temperature) from a smartphone were used to evaluate occupational heat stress produced by a hot work environment, the results suggest that this type of system is more efficient, discreet and less costly than using standard methods. As well it allows workers to take care of their health based on objective information and warnings. Similar to other studies, it concludes that including new sensors and devices, such as wristbands can offer new opportunities for preventing and monitoring health.

\subsubsection{Light}

An initiative regarding exposure to physical agents was made by (Cerqueira et al. 2017) where the accuracy of smartphones is reported using different applications for the measurement of light exposure with a focus on occupational health. Showing that smartphones have limitations and variability and may not be considered useful in occupational lighting assessments. 


\subsection{Wearables for assessment of exposure to chemical agents.}

A study made by (Negi et al. 2011) evaluated the exposure of chemical agents through the use of an adhoc wearable device with sensors for measuring hydrocarbons, total acids, humidity, and temperature. This system can monitor and detect gases (toxic hydrocarbons and acid environments) and can be applied to occupational and environmental health. This study sets the paradigm of continuous monitoring in real-time and shows that it is possible to use a smartphone as a user interface for the sensor device.

\subsection{Wearables for location of potential hazards (moving machinery)}

Another approach to safety was presented by (Ferreira et al. 2017) in which wearable devices were placed on worker safety vests and arms. The devices were used as an alarm (visual and sound) to alert maintenance workers near train tracks the proximity of a train. This study identified the challenges that need to be addressed to present a plausible technological security solution for railway personnel involved in maintenance work, in which includes the wireless communication and usability related to worker's perceptions of the alerts.

\section{DISCUSSION \& CONCLUSIONS}

Based on the literature review, a growing number of publications that use wearable devices for occupational risk assessment have been identified. The solutions use both commercial and specific wearable devices.

In the analyzed studies, it has been identified the use of devices developed specifically for particular risk assessment tasks as well as a growing number of solutions that use smart devices mainly smartphones and smartwatches, but some of these solutions only use one or more sensors and do not take advantage of their communication capabilities.

Several applications of this kind of technology for occupational risk assessment have been identified, among which are:

- Motion and activity detection.

- Detection of risk factors related to the musculoskeletal system.

- Fall detection and prevention.

- Exposure to physical and chemical agents (noise, vibration, gases, light).

- Location of potential hazards (moving machinery).

Most of this wearable solutions are focused on monitoring and alert (visual or haptic feedback), but with the support of other technologies or devices (e.g., smartphones or tablets) they could make use of their characteristics for making a complete system for management, training, and workers' awareness.

Despite the fact that the adoption of wearable devices is beginning and that many innovative solutions have been presented for the management of specific occupational risks, the integration of this solutions is not yet complete due to the lack of a common framework for development and future needs. A framework can make use of the features and advantages provided by the information and communications technologies and data analysis (based for example on the Big Data paradigm).

Also, the accuracy and reliability of these systems must be taken into account when they are used as a measurement system. At present, the built-in sensors of some intelligent devices are less precise than precision equipment, which makes difficult to perform a correct risk assessment. However, these devices could be used to obtain measurements with better representativeness because they can be worn in a continuous manner over the time.

In the future, it is expected to use the IoT approach in sensors, tools, and workers to improve the management of occupational safety. The first experiences in the use of wearables as integral management systems have been detected (Podgorski et al. 2017, Bernal et al. 2017, Li 2018), in which include the use of ad-hock wearable devices or personal protective equipment with sensors. Showing the need for innovative solutions that integrate monitoring, management, and workers training. Taking into account the diversity of risks that can be found in the workspace, as well as the real training and education needs of workers in different industries.

\section{REFERENCES}

Bernal, G., Colombo, S., Al Ai Baky, M. \& Casalegno, F. 2017, "Safety: Designing IoT and Wearable Systems for Industrial Safety through a User Centered Design Approach", Proceedings of the 10th International Conference on Pervasive Technologies Related to Assistive Environments ACM, 163.

Casilari, E., Luque, R. \& Moron, M. 2015, "Analysis of Android Device-Based Solutions for Fall Detection", Sensors, vol. 15, no. 8, 17827-17894.

Cerqueira, D., Carvalho, F. \& Melo, R.B. 2017, "Is It Smart to Use smartphones to measure illuminance for occupational Health and Safety Purposes?” International Conference on Applied Human Factors and Ergonomics Springer, 258.

Chan, M., Estève, D., Fourniols, J., Escriba, C. \& Campo, E. 2012, "Smart wearable systems: Current status and future challenges", Artificial Intelligence in Medicine, vol. 56, no. 3, 137-156.

Cutini, M. \& Bisaglia, C. 2014, "Whole body vibration monitoring using a smartphone", Ageng 2014 Zurich; International Conference of Agricultural (EurAgEng), 8.

Daponte, P., De Vito, L., Picariello, F. \& Riccio, M. 2013, "State of the art and future developments of measurement applications on smartphones", Measurement: Journal of the 
International Measurement Confederation, vol. 46, no. 9, 3291-3307.

Ding, L.Y., Zhou, C., Deng, Q.X., Luo, H.B., Ye, X.W., Ni, Y.Q. \& Guo, P. 2013, "Real-time safety early warning system for cross passage construction in Yangtze Riverbed Metro Tunnel based on the internet of things", Automation in Construction, vol. 36, 25-37.

Dumoulin, R. \& Voix, J. 2013, "Calibration of smartphonebased devices for noise exposure monitoring: Method, implementation, and uncertainties of measurement", The Journal of the Acoustical Society of America, vol. 133, no. 5, 3317-3317.

EU OSHA 2017, Monitoring technology: the 21st century's Pursuit of well-being?

Ferreira, B, et al., C. 2017, "Wearable computing for railway environments: proposal and evaluation of a safety solution", IET Intelligent transport systems, vol. 11, no. 6, 319-325.

Gjoreski, M., Gjoreski, H., Lustrek, M. \& Gams, M. 2016, "How Accurately Can Your Wrist Device Recognize Daily Activities and Detect Falls?", Sensors, vol. 16, no. 6, 800.

Habib, M., Mohktar, M., Kamaruzzaman, S., Lim, K., Pin, T. \& Ibrahim, F. 2014, "Smartphone-Based Solutions for Fall Detection and Prevention: Challenges and Open Issues," Sensors, vol. 14, no. 4, 7181-7208.

Hämäläinen, P., Takala, J. \& Kiat, T.B. 2017, Global estimates of occupational Accidents and work-related illnesses 2017, Workplace Safety and Health Institute.

Kardous, C. \& Shaw, P. 2014, "Evaluation of smartphone sound measurement applications", Journal of the Acoustical Society of America, vol. 135, no. 4, EL186-EL192.

Kardous, C.A. \& Celestina, M. 2015, "Use of smartphone sound measurement apps for occupational noise assessments", Journal of the Acoustical Society of America, vol. 137, no. 4, 2292.

Kardous, C.A. \& Shaw, P.B. 2016, "Evaluation of smartphone sound measurement applications (apps) using external microphones-A follow-up study", The Journal of the Acoustical Society of America, vol. 140, no. 4, EL327-EL333.

Kim, S. \& Nussbaum, M. 2013, "Performance evaluation of a wearable inertial motion capture system for capturing physical exposures during manual material handling tasks", Ergonomics, vol. 56, no. 2, 314-326.

Kim, K. \& Shin, D. 2015, "An acceptance model for smartwatches Implications for the adoption of future wearable technology", Internet Research, vol. 25, no. 4, 527-541.

Labrador, M.A. \& Lara Yejas, O.D. 2013, Human activity recognition: using wearable sensors and smartphones, 1st EDN, Taylor \& Francis, Boca Raton.

Lee, W., Lin, K., Seto, E. \& Migliaccio, G. 2017, "Wearable sensors for monitoring on-duty and off-duty worker physiological status and activities in construction", Automation in Construction, vol. 83, 341-353.

Li, R.Y.M. 2018, "Smart Working Environments Using the Internet of Things and Construction Site Safety" in An Economic Analysis on Automated Construction Safety 137-153.

Liu, B. \& Koc, A.B. 2013, "Hand-Arm Vibration Measurements and Analysis Using Smartphones", 2013 Kansas City, Missouri, July 21-July 24, 2013American Society of Agricultural and Biological Engineers, 1.

Matthies, D., Bieber, G. \& Kaulbars, U. 2016, "AGIS: automated tool detection \& hand-arm vibration estimation using an unmodified smartwatch", ACM, 1.

Mortazavi, B., Nemati, E., VanderWall, K., Flores-Rodriguez, H., Cai, J., Lucier, J., Naeim, A. \& Sarrafzadeh, M. 2015, "Can Smartwatches Replace Smartphones for Posture Tracking?", Sensors, vol. 15, no. 10, 26783-26800.

Murphy, E. \& King, E. 2016, "Testing the accuracy of smartphones and sound level meter applications for measur- ing environmental noise", Applied Acoustics, vol. 106, 1622.

Nast, D., Speer, W. \& Le Prell, C. 2014, "Sound level measurements using smartphone "apps": Useful or inaccurate?" NOISE \& HEALTH, vol. 16, no. 72, 251-256.

Nath, N., Akhavian, R. \& Behzadan, A. 2017, "Ergonomic analysis of construction worker's body postures using wearable mobile sensors", Applied Ergonomics, vol. 62, 107117.

Negi, I., et al., 2011, "Novel monitor paradigm for real-time exposure assessment", Journal of Exposure Science and Environmental Epidemiology, vol. 21, no. 4, 419-426.

Page, T. 2015, "A Forecast of the Adoption of Wearable Technology", International Journal of Technology Diffusion, vol. 6, no. 2, 12-29.

Pancardo, P., Acosta, F., Hernandez-Nolasco, J., Wister, M. \& Lopez-de-Ipina, D. 2015, "Real-Time Personalized Monitoring to Estimate Occupational Heat Stress in Ambient Assisted Working", Sensors, vol. 15, no. 7, 16956-16980.

Pannurat, N., Thiemjarus, S. \& Nantajeewarawat, E. 2014, "Automatic Fall Monitoring: A Review", Sensors, vol. 14, no. 7, 12900-12936.

Peppoloni, L., Filippeschi, A., Ruffaldi, E. \& Avizzano, C. 2016, "(WMSDs issue) A novel wearable system for the online assessment of risk for biomechanical load in repetitive efforts", International Journal of Industrial Ergonomics, vol. 52, 1-11.

Podgorski, D., Majchrzycka, K., Dabrowska, A., Gralewicz, G. \& Okrasa, M. 2017, "Towards a conceptual framework of OSH risk management in smart working environments based on smart PPE, ambient intelligence and the Internet of Things technologies", International Journal of Occupational Safety and Ergonomics, vol. 23, no. 1, 1-20.

Ramos, F., Moreira, A., Costa, A., Rolim, R., Almeida, H. \& Perkusich, A. 2016, "Combining smartphone and smartwatch sensor data in activity recognition approaches: An experimental evaluation", 267.

Sazonov, E., Neuman, M.R. 2014, Wearable sensors: fundamentals, implementation and applications, Academic Press.

Tarabini, M., Saggin, B., Scaccabarozzi, D. \& Moschioni, G. 2012, "The potential of micro-electro-mechanical accelerometers in human vibration measurements", Journal of Sound and Vibration, vol. 331, no. 2, 487-499.

Valero, E., Sivanathan, A., Bosche, F. \& Abdel-Wahab, M. 2017, "Analysis of construction trade worker body motions using a wearable and wireless motion sensor network", Automation in Construction, vol. 83, 48-55.

Wolfgang, R. \& Burgess-Limerick, R. 2014, "Using Consumer Electronic Devices to Estimate Whole-Body Vibration Exposure", Journal of Occupational and Environmental Hygiene, vol. 11, no. 6, D77-D81.

Wolfgang, R., Di Corleto, L. \& Burgess-Limerick, R. 2014, "Can an iPod Touch Be Used to Assess Whole-Body Vibration Associated with Mining Equipment?", Annals of Occupational Hygiene, vol. 58, no. 9, 1200-1204.

Wright, R. \& Keith, L. 2014, "Wearable Technology: If the Tech Fits, Wear It", Journal of Electronic Resources in Medical Libraries, vol. 11, no. 4, 204-216.

Yan, X., Li, H., Li, A. \& Zhang, H. 2017, "Wearable IMUbased real-time motion warning system for construction workers' musculoskeletal disorders prevention", Automation in Construction, vol. 74, 2-11.

Yang, C. \& Hsu, Y. 2010, "A review of accelerometry-based wearable motion detectors for physical activity monitoring", Sensors, vol. 10, no. 8, 7772-7788.

Zhu, Y., Li, J., Liu, L. \& Tham, C. 2015; 2016, "iCal: Intervention-free Calibration for Measuring Noise with Smartphones", 85. 